\title{
RESEARCH AND ENGINEERING PRACTICE OF EMERGENCY GEOGRAPHIC INFORMATION COLLABORATIVE SERVICE MODE WITH CROWDSOURCED DATA
}

\author{
Yang Jing ${ }^{1, *}$, Huang $\mathrm{Wei}^{1}$,Zhang Hongping ${ }^{1}$, Li Heng $^{1}$ \\ National Geomatics Centre of China, Beijing, China- (yangjing, huangwei, zhanghongping)@ngcc.cn
}

Commission IV, WG IV/6

KEY WORDS: Crowdsourcing, Volunteered Geographic Information, Disaster Emergency Management, Data Mining, Collaborative Services, MAPWORLD.

\begin{abstract}
:
China is a disaster prone country. In recent years, the national functional departments have been committed to the construction of emergency surveying and mapping support capacity. However, the data owned by government departments are often systematic, accurate and authoritative, but the real-time is not enough. The improvement of data update efficiency requires a lot of resources. The main body of emergency management is also mainly government departments, which lacks public participation and interaction. The application of volunteer geographic information in China's emergency management has not yet formed a scale. This article will mainly studies the mechanism of volunteer information in emergency management, the multi-source emergency geographic information collaborative service mode with volunteer participation, and take specific engineering practices as an example to introduce the national Geographic Information Public Service Platform for Disaster Emergency, and try to solve the problems about real-time disaster site information acquisition, multi-source information online integration and collaborative services, and rapid customization and release of user-oriented emergency topics in multiple emergency scenarios Issues.
\end{abstract}

\section{INTRODUCTION}

China is a country prone to natural disasters and those disasters bring huge losses to the industrial and agricultural production of China and people's lives here every year. For a long time, the main participants in disaster emergency management have been the government and its departments, social organizations and the military (Feng K., 2020), while the power that the public can exert is very limited. However, Obtaining practical and guiding map data and real-time disaster information especially multimedia information such as pictures, videos etc. at the disaster site in a short period of time is very important for the government to organize rescue and the public to understand the disaster information. The "Crisis mappers", who played an important role in the Nepal earthquake disaster relief operation, made people realize that the research on the crowdsourcing model in disaster relief response is worthy of in-depth exploration and practice so as to allow volunteers to play more in disaster release, disaster relief, post-disaster reconstruction, etc. (Ahmed. A, 2018, Li, L., 2012).

This article will mainly studies the mechanism of volunteer information in emergency management, the multi-source emergency geographic information collaborative service mode with volunteer participation, and take specific engineering practices as an example to introduce the national Geographic Information Public Service Platform for Disaster Emergency, and try to solve the problems about real-time disaster site information acquisition, multi-source information online integration and collaborative services, and rapid customization and release of user-oriented emergency topics in multiple emergency scenarios Issues.

\section{APPLICATION OF CROWDSOURCING INFORMATION IN EMERGENCY MANAGEMENT AND SERVICE PLATFORM CONSTRUCTION}

\subsection{Application of crowdsourcing information in emergency management}

Crowdsourcing was first proposed by J. Howe in the June 2006 issue of Wired magazine in the United States (HOWE J., 2006) .It is defined as a company or organization outsources work tasks performed by past employees in the form of free resources to non-specific (usually large-scale) ) The practice of the public network". Some scholars believe that the motivation of crowdsourcing is to reduce costs, solve problems, and seek ideas (Xiao, 2012), the essence is an open innovation, the driving force is the social needs of volunteers, while the realization carrier is the network (Sun, 2012).

In recent years, there have been more and more successful cases of emergency management in dealing with emergencies through crowdsourcing, and the application methods have become more extensive. For example, after the 2010 Haiti earthquake, a variety of public participated in emergency management, completed real-time map drawing of the disaster area, sent and processed short messages for victims' needs, etc., which provided assistance to the rescue to a large extent (Ziemke, J. , 2012) In China, The stopped Beijing International Trade Light

* Corresponding author 
Show and the Shanghai Bund stampede are happened on the same day(December 31, 2014). The thermal image of the on-site human flow monitored by Tencent's WeChat can intuitively determine the intensity of the flow of people at that time. The reason for the different results of the two incidents lies in whether the regulatory authorities captured relevant information provided by the public in a timely manner. The former handled the activities quickly and avoided the occurrence of emergencies. It can be seen that paying attention to the excavation and utilization of public participation information is very important for the emergency management of government departments.

Furthermore, some scholars began to study the application mechanism and problems of crowdsourcing information in emergency management. Some studies have pointed out that applying the crowdsourcing model to the emergency management process can bring more extensive participation in the collection and verification of information and ships. The process is mainly divided into publishing information, capturing information, processing and analyzing information, and issuing instructions (Chen, 2016). At the same time, some authors also pointed out that although the volunteered information can be a reliable resources for disaster management, the issues of data quality, bias in contributions, data management, and the security of individuals, authorities and their information need to be researched and solved deeply (Haworth, 2015). Moreover, Wang Qian and Dai Jiaxin introduced a new model of government outsource, public insource, and joint participation in government governance (Wang and Dai, 2014), this method can be used as an effective reference for the collaboration between the government and the public in emergency management.

\subsection{Application of crowdsourcing information in emergency service platform construction}

The mode of data acquisition using VGI mode has been relatively mature in foreign countries, and most of them get, manage (Li and Goodchild, 2006) and share disaster information in the form of network community. Toshinari Nagasaka (Nagasaka T., 2006) has developed a network community platform for disaster response based on VGI, which allows all residents to edit various data on the platform after the disaster, so as to realize the data sharing between the government and residents, residents and residents, and has been successfully applied to the station disasters in Fujisawa city and Shimada City, Japan. Ushahidi platform developed by Meier P. (Meier P., 2012 and 2011) was successfully applied to Haiti earthquake on January 12, 2010, Japan earthquake on March 2011 and Russia fire in summer 2012, which also integrates real-time information and multimedia data of disaster scene based on VGI idea.

Although there is a growing research body concerned with the analysis of the usage of social media during disasters, most previous work has concentrated on using social media as a standalone information source, whereas its combination with other information sources holds a still underexplored potential (De Albuquerque, etc., 2015). At present, there are few researches on the use of VGI mode for emergency data acquisition in China, but there are many scholars using mobile GIS correspondence and data acquisition, such as disaster data acquisition system designed by Liu Rui (Liu, 2012), water pollution data processing system designed by Tan Xingbin (Tan, 2011.), and Taipei urban emergency system designed by Wei Youbing (Wei, 2007) using PDA.

It is obviously that the application of VGI in China's geographic information, especially in emergency relief and public service, needs further standardization and in-depth development. The national Geographic Information Public Service Platform for Disaster Emergency urgently needs the addition of VGI information. On the one hand, it can improve the richness and timeliness of data, on the other hand, it can provide users with broader application scenarios.

\section{MULTI-SOURCE EMERGENCY GEOGRAPHIC INFORMATION COLLABORATIVE SERVICE MODE WITH VOLUNTEER PARTICIPATION}

\subsection{Information providers and their effect}

The providers of crowdsourcing information in emergency management mainly include the public affected by the incident, the public on social networks, and data volunteers (CHEN Jing, 2016). These three types of providers supply basic data for disaster emergency management through the level-by-level release, utilization and circulation of information, forming a collaborative mechanism for crowdsourcing information in emergency management.

Specifically, the main body of crowdsourcing information includes the affected people, social network public, data volunteers and relevant government departments. They play different roles in the emergency management task:

The public affected is directly affected by the emergency, providing the most intuitive event information, including time, place, disaster degree and impact. Through mobile positioning devices and networks, they provide and share the local intuition about the impact of the disaster event at the time, helping government departments and the public to better understand and judge the hazard situation at the disaster site. On the other hand, they can also provide timely feedback, the needs of disaster relief, such as food, water, rescue force, etc., provide the most direct basis for disaster relief work.

Social network public can quickly obtain and provide diversified emergency information through social network. Based on their trust in each other, they interact and communicate with the affected public through the Internet, and then seek additional help from the society. They often bring together a variety of knowledge backgrounds, and sometimes bring unexpected professional knowledge related to emergency.

Data volunteers refer to volunteers who participate in incident handling after a disaster event. Although they are physically scattered, they tend to improve the efficiency of online collaboration through some open source methods and provide open data required for emergency management, including volunteer geography. Information data. Their main role is to be able to process and analyze emergency information and provide structured information for the emergency management platform.

Relevant departments are the direct organizers and responsible persons of the national emergency prevention and monitoring, post-disaster rescue and reconstruction work. They have more professional resources and power, but the data they have and the information provided by social forces are usually different, sometimes even lagging behind those information obtained by social forces. Therefore, the effective use of crowdsourcing and volunteer information is very important for government departments to obtain information effectively, obtain 
information in time, and make scientific and reasonable decisions.

\subsection{Multi-participant emergency information collaborative} service model

In recent years, several government departments have established emergency public service platforms to provide the society with disaster warning and rescue information release. However, most of these platforms obtain information based on their own capabilities, and lack effective integration and utilization of crowdsourced data and volunteer information. The author believes that the multi-source emergency information collaborative service model involving volunteers should be like this: the public affected by the incident can provide intuitive information on the disaster site, which is submitted to the social network, and the social network public obtains, extracts, and combines their own knowledge After editing and publishing, data volunteers will provide structured data to the emergency public service platform after processing and analysis. Those platform integrates and analyzes the cleaned volunteer information with various types of thematic information such as transportation, water conservancy and social governance, so as to provide the government and the public with effective emergency management information that can support decisionmaking. By obtaining effective emergency management information, government functional departments can make command decisions and social services, and then command all forces to carry out disaster relief and post disaster reconstruction.

Significantly, the disaster emergency management related functional departments will also release authoritative information, including disaster warning information, emergency event information, handling and rescue information, and recovery and reconstruction information. However, most of these information lack spatial characteristics and are not convenient for intuitive understanding and comprehensive judgment. In this situation, basic geographic information with spatial attributes is requires (Kemper, H. and Kemper, G., 2020).

Geographical information is the link connecting multi-source emergency information, an important carrier for information integration and collaborative services, and also important basic information for comprehensive analysis and disaster research and judgment. Crowdsourced information, authoritative disaster emergency management information, and basic geographic information can be used through a series of integrated and utilized strategies to form disaster emergency geographic information that can be expressed online. Through visual expression and spatial analysis strategies, collaborative services can be realized to meet the public's requirements for disaster early warning, disaster information acquisition, analysis and utilization.

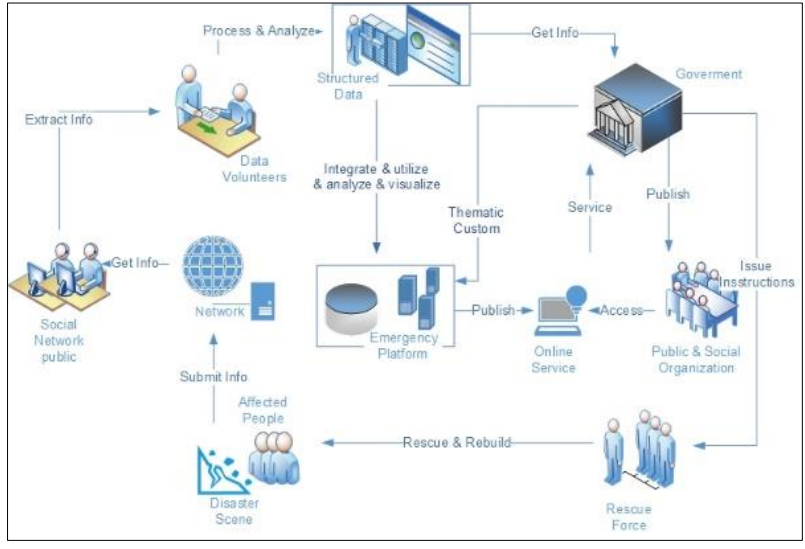

Figure 1. Multi-source emergency geographic information collaborative service mode with volunteer participation

\section{CONSTRUCTION PRACTICE OF THE NATIONAL GEOGRAPHIC INFORMATION PUBLIC SERVICE PLATFORM FOR DISASTER EMERGENCY BASED ON VOLUNTEER INFORMATION}

\subsection{Overview}

The National Geographic Information Public Service Platform for Disaster Emergency is an important task in the construction of China's emergency surveying and mapping support capacity. Its construction purpose is to release emergency basic geographic information and disaster information rapidly to the public based on the Internet, and to provide services for the public; to establish special release and demonstration system on online and establish special issue and demonstration system facing the needs of media publicity (Yang, 2019).

The National Geographic Information Public Service Platform for Disaster Emergency mainly relies on the existing geographic information public service resources and support capacity, combined with the actual needs of Surveying and mapping support work, integrates and publishes emergency basic information, emergency thematic information and emergency event information. On the basis of professional information collection, VGI provides real-time disaster location, disaster description and rescue information from the public at the scene of emergency, which can greatly improve the timeliness of information release of public service platform. At the same time, considering that this kind of information collection and release is quite different from professional surveying and mapping geographic information, the design of public service platform must take into account the collection, integration and utilization of this special information source.

The approach is to aggregate volunteer information and ubiquitous network resources intelligently to build a comprehensive information system for emergency management based on national geographic information public service. On the one hand, the platform need to solve the problem of lack or overload of information before, during and after disasters, and on the other hand, it must allow the authoritative departments to do spatial analysis, comprehensive research and judgment, customized thematic information release, etc. 


\subsection{Crowdsourcing Data Collection, Utilization and Release}

\section{Process on the Platform}

The main feature of the platform is the integration of volunteer information, expanding the scope of information collection and enhancing the timeliness of information acquisition. Security and data quality are major concerns for VGI (Hongping zh., 2012). However, volunteer information is mostly spontaneous contribution data, and most of them are non-professionals, so the data quality is unpredictable, and the continuity and uniformity cannot be guaranteed (Shan, etc., 2014). The collection and utilization process of volunteer information mainly includes data model establishment, information collection, data cleaning, visual expression (Pu and Wang, 2008) and analysis \& mining (Figure 2).

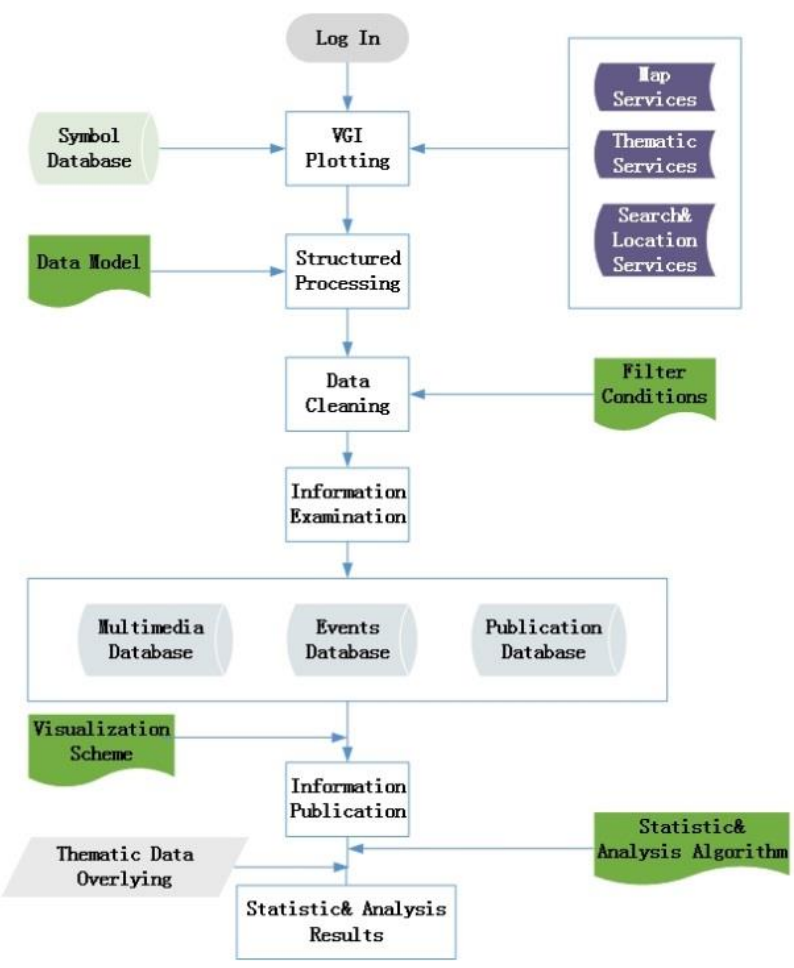

Figure 2. Crowdsourcing information utilization process on the platform

Data Modelling: In order to ensure the accurate availability of crowdsourcing information, it is necessary to first build a unified information collection model according to the needs of information integration and utilization, determine the data content, field types and restrictions in advance, and collect the necessary and structured emergency information from the information providers in the form to ensure the maximum availability of the collected data.

Data Acquisition: Develop the emergency event plotting function, provide a unified event plotting symbol library and information collection form to collect and express all kinds of emergency events and on-site information. At the same time, users can accurately locate the plotting points based on the base map provided by the station, combined with the query and search function. Considering the uncertainty of the source of Internet users, in order to prevent malicious plotting, users who log on to the national emergency surveying and mapping public service platform must be based on unified user real name authentication to ensure the stability and legitimacy of the information source to the greatest extent.

Data Cleaning: Considering the unpredictable quality of crowdsourced data, in order to achieve the purpose of authoritative publishing, it is necessary to clean and audit the data. Using mature professional data processing tools, and using certain data cleaning principles, the data logic is checked to ensure that the plotting content is consistent with the actual location. At the same time, the duplicate data, illegal content and other "dirty data" that do not meet the collection and release requirements are removed, and the cleaned data can enter the audit process. At the same time, the necessary manual audit link is set to verify and filter the event information from the perspective of information expression and public release requirements. On this basis, for the government or professional users to implement audit free, no need to go through the background audit, directly release authoritative information, to ensure the rapid release of real-time information.

Data Visualization: After examination and verify, the volunteer information is released on the platform in the form of emergency. Due to the wide range of information sources provided by volunteers, but the coverage area is extremely uneven, it is difficult to achieve uniform coverage. At the same time, massive volunteer data cannot be clearly expressed in the limited field of vision, so the point clustering algorithm based on grid and distance is used in the visualization, and the number of target points in a certain statistical unit is taken as the expression object to improve the visualization effect. At the same time, the event information list and certain filtering function are used to ensure the accurate positioning of specific events.

Data Analysis and Mining: The volunteer geographic information provided by crowdsourcing users contains rich social attributes, semantic information and temporal information, so it is necessary to realize more knowledge discovery and extraction through necessary analysis and mining means. The function of statistical analysis within and across layers is developed to analyze the temporal and spatial distribution law and aggregation degree of emergency disaster events. By overlaying with emergency rescue information such as rescue infrastructure and emergency shelter, the relevant knowledge of disaster analysis and rescue command can be obtained. By overlaying with terrain, road traffic and other information, it can provide reference for emergency rescue, rescue and rescue It can provide reference for disaster prevention and reduction.

\subsection{Platform Architecture}

The Platform is consist of Infrastructure Layer, Data Layer, Service Layer, Application Layer, and Presentation layer, and will support volunteer information collection, data release and management, information query, spatial analysis, online mapping, customized topic construction, release and sharing, etc.

Infrastructure Layer: The Infrastructure Layer is the external foundation of the system construction, which provides the hardware environment, software environment and network environment for the system construction, and is the necessary factor for the system operation, data storage, network communication and other links. The hardware environment includes data server, application server, storage device, mobile terminal, front-end acquisition device, office computer, etc.; the software environment includes operating system, database software, support environment software, browser and plug-in, etc.; the network environment includes machine room, router, 
firewall, broadband service, etc.

Data Layer: The Data Layer mainly stores basic geographic information of Surveying and mapping, emergency thematic information and emergency volunteer geographic information through relational and non-relational databases. The basic geographic information of Surveying and mapping includes the geographic information data of Surveying and mapping at all levels and the service data of sky map; the emergency thematic information includes the infrastructure data obtained from the extraction, integration and utilization of the basic geographic information, as well as the emergency thematic data of authoritative departments obtained through the network means and dynamically stored and distributed; the emergency volunteer geographic information is mainly based on the voluntary information of volunteers Plotting and uploading emergency points and their descriptive information.

Service Layer: The service layer is not only the link between data and application, but also the basic unit of function module, which provides component and modular function support for application layer. The platform mainly includes two types of services: Geographic services based on OGC standard and web application services based on W3C standard. Basic services include tile map services, multi temporal services, etc; Application services mainly realize functional applications, including place name search service, path planning, (reverse) geocoding, administrative division and other services; Vector services are OGC annotation services, vector rendering services and emergency thematic layers based on background vector data; Special service is the application object to realize the service functions of plotting, statistical analysis and web sharing.

Application Layer: The Application Layer is oriented to the application needs of all kinds of users and provides application processing and information services. The micro-service development model based on typical separation of front end and back end is adopted. Each module follows the principle of "high cohesion and low coupling", and the idea of "divide and rule" is adopted. The key problems to be solved in each module are divided and solved separately, which is easy to control, extend and allocate resources. It mainly includes element content processing, positioning accuracy processing, resource registration, resource query positioning, website dynamic creation and management, emergency thematic information map display, emergency thematic information overlay and layer management, emergency information query, emergency information statistics, emergency information analysis, image comparative analysis, emergency event plotting and management, multimedia information management and user management It has many application functions.

Presentation layer: The Presentation layer is a part of the system that directly interacts with users, including its presentation carrier and presentation form. The forms of expression include map positioning, data management, statistical charts, service management, data display, thematic map, etc.

Users: Users include people or organizations related to the platform. Ordinary users can query and browse information based on the platform, and stack all kinds of services for statistical analysis; the registered users of the system can plot and upload the emergency information based on the common user rights according to the user type, and they are the main contributors of the information. At the same time, they can carry out the superposition analysis of a variety of emergency information based on various services provided by the platform, and then customize and share the emergency special webpage.

The completed National Geographic Information Public Service Platform is shown in the figure below.

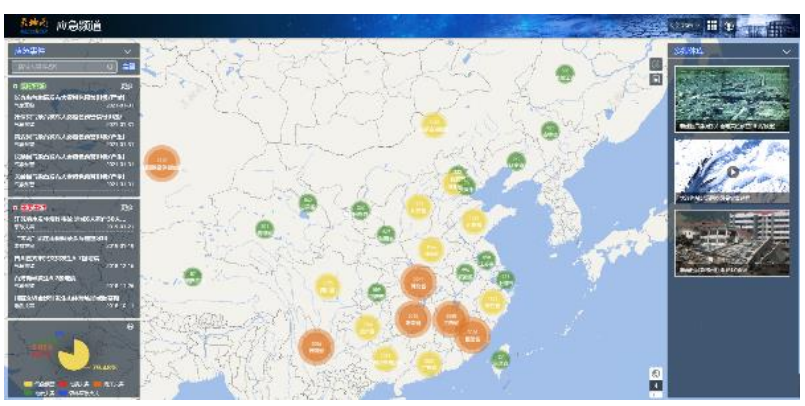

Figure 3. National Geographic Information Public Service Platform for Disaster Emergency

\section{CONCLUSION}

The engineering practice results were released on the National Platform of Common Geospatial Information Services, providing service support for the government, emergency management departments and the public. It has successively supported the emergency management decision-making and public information popularization of a series of disasters, such as the collapse of mountain in Mao Xian County, the Jiuzhaigou earthquake, the explosion of chemical enterprises in Jiangsu Province, and the flood disaster in China, 2020.

In conclusion, through the effective utilization of volunteer we can solve the problems of lack or overload of information, data outdate and low update efficiency at all stages of emergency management to a certain extent; Effective integration and utilization of volunteer information and ubiquitous network information resources based on geographic information public service can provide reliable decision-making basis for emergency management; The national Geographic Information Public Service Platform for Disaster Emergency can release authoritative, accurate and timely emergency basic geographic information and disaster thematic information to provide online services for the public, And then support the comprehensive decision-making and social services of government departments.

\section{ACKNOWLEDGEMENTS}

The work was supported by National Key Research and Development Program of China (NO.2017YFB05033705).

\section{REFERENCES}

Feng, K., 2020: Understanding government's role in integrated disaster risk governance. Journal of Public Affairs.

Ahmed, A., Hochmair, H. H., \& Sreten, C., 2018: Analyzing the effect of earthquakes on openstreetmap contribution patterns and tweeting activities. Geo Spatial Information Science, 1-18.

Li, L., \& Goodchild, M. F., 2012: The role of social networks in emergency management: a research agenda. International 
Journal of Information Systems for Crisis Response \& Management, 2(4), 48-58.

HOWE J., 2006: The rise of crowdsourcing. Wired Magazine, $14(6): 1-4$

Xiao L., Gao C.C., 2019: Crowdsourcing changes the innovation model of enterprises. Shanghai Economic Research, (9): 35-41.

Sun J., Song F.G., Chen H.L., 2012: Discussion on the construction of the navigation library system based on the crowdsourcing model. Library Journal, (9): 38-41.

Ziemke, J., \& Liu, S. B., 2012: From Cultures of Participation to the Rise of Crisis Mapping in a Networked World. The Participatory Cultures Handbook, 185-196.

Chen J., 2016: Research on Collaboration Mechanism of Information Agent in Emergency Management Based on Crowdsourcing. Information Studies: Theory \& Application, 039(005):69-73.

Haworth, B., \& Bruce, E., 2015: A review of volunteered geographic information for disaster management. Geography Compass, 9(5), 237-250.

Qian, W., Dai, J.X., 2014: Government Governance Network the generation, construction and utility of crowdsourcing mode. Journal of Public Management, 11(04): 61-70+141-142.

Kemper, H., \& Kemper, G., 2020: Sensor Fusion, GIS and AI Technologies for Disaster Management.

Li, L., \& Goodchild, M. F., 2006: The role of social networks in emergency management: a research agenda. International Journal of Information Systems for Crisis Response \& Management, 2(4), 48-58.

Nagasaka T., 2006: New mode of risk governance enhanced by an e-community platform. A better integrated management of disaster risks: Toward resilient society to emerging disaster risks in mega-cities. Terra, Tokyo, 89-107.

Meier P., 2012: Crisis Mapping in Action: How Open Source Software and Global Volunteer Networks Are Changing the World, One Map at a Time. Journal of Map \& Geography Libraries, 8(2):89-100.

Meier, P., 2011: New information technologies and their impact on the humanitarian sector. International Review of the Red Cross, 93(884):1239-1263.

De Albuquerque, J. P., Herfort, B., Brenning, A., \& Zipf, A., 2015: A Geographic Approach for Combining Social Media and Authoritative Data towards Identifying Useful Information for Disaster Management. International Journal of Geographical Information Science, 29(4), 667-689.

Liu, R., 2012: A Study on Disaster Data Collection System Based On Handheld Mobile Terminal. Shanghai Normal University, SHH, CN.

Tan, X.B., 2011: The Research of Data Acquisition System Key Technology for Water Pollution Based On Mobile GIS. Chongqing University.

Wei, Y.B., 2007: Bulletin of Surveying and Mapping. Bulletin of
Surveying and Mapping, (3).

Yang, J., 2019: Design of the Public Service Platform for National Emergency Surveying and Mapping Considering Volunteered Geographic Information. Bulletin of Surveying and Mapping, No.510 (09):155-159.

Zhang, H.P., 2012: Development and Application of Volunteered Geographic Information. Geomatics World, 2012, 000(004):67-71.

Shan J, etc., 2014: Methods of Crowd Sourcing Geographic Data Processing and Analysis. Geomatics and Information Science of Wuhan University, 39(4):390-396.

Pu, P.X., Wang, Y., 2008: Preliminary Research on the System Architecture of the Platform Integrated by Emergency Geographic. Geomatics World, 6(6):39-42. 